\title{
On the clique number of a strongly regular graph
}

\section{Gary R. W. Greaves*}

Division of Mathematical Sciences, School of Physical and Mathematical Sciences, Nanyang Technological University, 21 Nanyang Link, Singapore 637371, Singapore gary@ntu.edu.sg

\author{
Leonard H. Soicher \\ School of Mathematical Sciences, \\ Queen Mary University of London, \\ Mile End Road, London E1 4NS, UK \\ L.H.Soicher@qmul.ac.uk
}

Submitted: May 18, 2018; Accepted: Sep 23, 2018; Published: Oct 19, 2018

(C) The authors. Released under the CC BY-ND license (International 4.0).

\begin{abstract}
We determine new upper bounds for the clique numbers of strongly regular graphs in terms of their parameters. These bounds improve on the Delsarte bound for infinitely many feasible parameter tuples for strongly regular graphs, including infinitely many parameter tuples that correspond to Paley graphs.
\end{abstract}

Mathematics Subject Classifications: 05C69, 05E30

\section{Introduction}

The clique number $\omega(\Gamma)$ of a graph $\Gamma$ is defined to be the cardinality of a clique of maximum size in $\Gamma$. For a $k$-regular strongly regular graph with smallest eigenvalue $s<0$, Delsarte [12, Section 3.3.2] proved that $\omega(\Gamma) \leqslant\lfloor 1-k / s\rfloor$; we refer to this bound as the Delsarte bound. Therefore, since one can write $s$ in terms of the parameters of $\Gamma$, one can determine the Delsarte bound knowing only the parameters $(v, k, \lambda, \mu)$ of $\Gamma$. In this paper we determine new upper bounds for the clique numbers of strongly regular graphs in terms of their parameters. Our bounds improve on the Delsarte bound infinitely often.

Let $q=p^{k}$ be a power of a prime $p$ congruent to $1 \bmod 4$. A Paley graph has vertex set equal to the finite field $\mathbb{F}_{q}$, and two vertices $a$ and $b$ are adjacent if and only if $a-b$ is a nonzero square. For a Paley graph $\Gamma$ on $q$ vertices with $k$ even, it is well-known ${ }^{1}$

\footnotetext{
*The first author was supported by JSPS KAKENHI; grant number: 26.03903

${ }^{1}$ The elements of the subfield of size $\sqrt{q}$ form a clique $B$. Blokhuis [4] showed that every clique of size $\sqrt{q}$ is obtained from a linear transformation of $B$.
} 
that $\omega(\Gamma)=\sqrt{q}$, which corresponds to equality in the Delsarte bound. Bachoc et al. [1] recently considered the case when $\Gamma$ is a Paley graph on $q$ vertices with $k$ odd and, for certain such $q$, showed that $\omega(\Gamma) \leqslant\lfloor\sqrt{q}-1\rfloor$. This corresponds to an improvement to the Delsarte bound for these Paley graphs.

Here, working much more generally, given a strongly regular graph $\Gamma$ with parameters $(v, k, \lambda, \mu)$, we provide inequalities in terms of the parameters of $\Gamma$ that, when satisfied, guarantee that the clique number of $\Gamma$ is strictly less than the Delsarte bound. We show that these inequalities are satisfied by infinitely many feasible parameter tuples for strongly regular graphs and, in particular, are satisfied by infinitely many parameter tuples that correspond to Paley graphs. Our inequalities are obtained using what we call the "clique adjacency bound" (see Section 4), a bound defined by the second author [17]. We also show that the clique adjacency bound is always at most the Delsarte bound when applied to strongly regular graphs.

We have made use of computer algebra assistance (using Maple Version 18 [3] and its Groebner package) to perform the direct calculations needed to verify certain equalities used in our proofs. We remark that the total CPU time for all this verification on a desktop Linux PC was only about 0.16 seconds, and the total store used by Maple was 2.4MB.

The paper is organised as follows. In Section 2 we state our main results and in Section 3 we state some standard identities that we will use in our proofs. Section 4 contains the proofs of our main results. In Section 5 we examine the strength of the clique adjacency bound and in Section 6 we provide an illustrative example comparing certain bounds for the clique number of an edge-regular graph that is not necessarily strongly regular. Finally, we give an appendix in which we describe our symbolic computations.

\section{Definitions and main results}

A non-empty $k$-regular graph on $v$ vertices is called edge-regular if there exists a constant $\lambda$ such that every pair of adjacent vertices has precisely $\lambda$ common neighbours. The triple $(v, k, \lambda)$ is called the parameter tuple of such a graph. A strongly regular graph $\Gamma$ with parameter tuple $(v, k, \lambda, \mu)$ is defined to be a non-complete edge-regular graph with parameter tuple $(v, k, \lambda)$ such that every pair of distinct non-adjacent vertices has precisely $\mu$ common neighbours. We refer to the elements of the parameter tuple as the parameters of $\Gamma$. We call the parameter tuple of a strongly regular graph feasible if its elements satisfy the nonnegativity and divisibility constraints given by Brouwer $[6$, VII.11.5]); see the results of Section 3.

Let $\Gamma$ be a strongly regular graph with parameters $(v, k, \lambda, \mu)$. It is well-known that $\Gamma$ has at most three distinct eigenvalues, and moreover, the eigenvalues can be written in terms of the parameters of $\Gamma$ (see Cameron and Van Lint [9]). In what follows we denote the eigenvalues of $\Gamma$ as $k \geqslant r>s$.

Strongly regular graphs whose parameters satisfy $k=(v-1) / 2, \lambda=(v-5) / 4$, and $\mu=(v-1) / 4$ are called type I or conference graphs. Strongly regular graphs all of whose eigenvalues are integers are called type II. Every strongly regular graph is either 
type I, type II, or both type I and type II (see Cameron and Van Lint [9, Chapter 2]).

The fractional part of a real number $a \in \mathbb{R}$ is defined as frac $(a):=a-\lfloor a\rfloor$. We are now ready to state our main results.

Theorem 1. Let $\Gamma$ be a type-I strongly regular graph with $v$ vertices. Suppose that

$$
0<\operatorname{frac}(\sqrt{v} / 2)<1 / 4-(\sqrt{v+5 / 4}-\sqrt{v}) / 2 .
$$

Then $\omega(\Gamma) \leqslant\lfloor\sqrt{v}-1\rfloor$.

Proof. Follows from Theorem 11 together with Corollary 16 below.

Remark 2. For a prime $p$ satisfying $0<\operatorname{frac}(\sqrt{p} / 2)<1 / 4-(\sqrt{p+5 / 4}-\sqrt{p}) / 2$, we have that $\lfloor\sqrt{p}\rfloor=2\lfloor\sqrt{p} / 2\rfloor$ is even. Furthermore, for $n:=\lfloor\sqrt{p}\rfloor$, since $\lfloor\sqrt{p}\rfloor>\sqrt{p+5 / 4}-1 / 2$, we have $n^{2}+n-1>p$. Hence, if $\Gamma$ is a Paley graph on $p$ vertices then $\omega(\Gamma) \leqslant\lfloor\sqrt{p}-1\rfloor$ by Bachoc et al. [1, Theorem 2.1 (i)] (see [1, Remark 2.5]). Therefore, for type-I strongly regular graphs with $p$ (a prime) vertices, satisfying $0<\operatorname{frac}(\sqrt{p} / 2)<1 / 4-(\sqrt{p+5 / 4}-$ $\sqrt{p}) / 2$, Theorem 1 is a generalisation of Bachoc et al. [1, Theorem 2.1 (i)].

Remark 3. Let $g$ be a positive integer. Then $(1+4 g, 2 g, g-1, g)$ is a feasible parameter tuple for a type-I strongly regular graph on $v=1+4 g$ vertices. Observe that $(\sqrt{v+5 / 4}-\sqrt{v}) / 2$ tends to 0 as $v$ tends to infinity. Using Fejér's theorem (see Kuipers and Niederreiter [15, page 13]), it is straightforward to show that the sequence $(\sqrt{1+4 g} / 2)_{g \in \mathbb{N}}$ is uniformly distributed modulo 1 . Therefore we can apply Theorem 1 to about a quarter of all feasible parameter tuples for type-I strongly regular graphs.

Let $\mathcal{P}$ denote the set of all primes $p$ of the form $p=1+4 g$ for some $g \in \mathbb{N}$. Then the sequence $(\sqrt{p} / 2)_{p \in \mathcal{P}}$ is uniformly distributed modulo 1 (see Balog [2, Theorem 1]). Therefore, since Paley graphs on $p$ vertices exist for all $p \in \mathcal{P}$, Theorem 1 is applicable to infinitely many strongly regular graphs.

Note that the example in [17] with parameters $(65,32,15,16)$ is an example of a (potential) graph satisfying the hypothesis of Theorem 1.

A graph is called co-connected if its complement is connected. Note that strongly regular graphs that are not co-connected are complete multipartite. We have the following:

Theorem 4. Let $\Gamma$ be a co-connected type-II strongly regular graph with parameters $(v, k, \lambda, \mu)$ and eigenvalues $k \geqslant r>s$. Suppose that

$$
0<\operatorname{frac}(-k / s)<1-\left(r^{2}+r\right) /(v-2 k+\lambda) .
$$

Then $\omega(\Gamma) \leqslant\lfloor-k / s\rfloor$.

Proof. Follows from Theorem 11 together with Corollary 19 below.

Remark 5. Currently Brouwer [7] lists the feasible parameter tuples for connected and co-connected strongly regular graphs on up to 1300 vertices. Of these, about $1 / 8$ of the parameter tuples of type-II strongly regular graphs satisfy the hypothesis of Theorem 4 . By the remark following Corollary 19, it follows that Theorem 4 can be applied to about $1 / 4$ of the complementary pairs of type-II strongly regular graphs on Brouwer's list. 
Note that the example in [17] of a strongly regular graph with parameter tuple $(144,39,6,12)$ is an example of a graph satisfying the hypothesis of Theorem 4 ; in fact, in this case, the conclusion of Theorem 4 is satisfied with equality. The parameter tuple $(88,27,6,9)$ is the first parameter tuple in Brouwer's list to which we can apply Theorem 4 and whose corresponding graphs are not yet known to exist (or not exist).

\section{$3 \quad$ Parameters of strongly regular graphs}

Here we state some well-known properties of strongly regular graphs and their parameters. The first two propositions are standard (see Brouwer and Haemers [8, Chapter 9] or Cameron and Van Lint [9, Chapter 2]).

Proposition 6. Let $\Gamma$ be a strongly regular graph with parameters $(v, k, \lambda, \mu)$ and eigenvalues $k \geqslant r>s$. Then

$$
\begin{aligned}
(v-k-1) \mu & =k(k-\lambda-1) ; \\
\lambda-\mu & =r+s \\
\mu-k & =r s
\end{aligned}
$$

Proposition 7. Let $\Gamma$ be a type-I strongly regular graph with parameters $(v, k, \lambda, \mu)$ and eigenvalues $k>r>s$. Then

$$
\begin{array}{r}
k=(v-1) / 2 ; \quad \lambda=(v-5) / 4 ; \quad \mu=(v-1) / 4 \\
r=(\sqrt{v}-1) / 2 ; \quad s=-(\sqrt{v}+1) / 2 .
\end{array}
$$

The next proposition is a key observation.

Proposition 8. Let $\Gamma$ be a strongly regular graph with parameters $(v, k, \lambda, \mu)$ and eigenvalues $k \geqslant r>s$.

(i) If $\Gamma$ is type I then $k / s-2 \operatorname{frac}(\mu / s)$ is an integer.

(ii) If $\Gamma$ is type II then $k / s-\operatorname{frac}(\mu / s)$ is an integer.

Proof. If $\Gamma$ is type I then, by Proposition 7 , we have $k-2 \mu=0$. If $\Gamma$ is type II then, by Proposition 6, we have $k / s-\mu / s=-r$ and $r$ is an integer.

Next, the complement $\bar{\Gamma}$ of a strongly regular graph $\Gamma$ is also a strongly regular graph. This is again a standard result (see Cameron and Van Lint [9, Chapter 2]).

Proposition 9. Let $\Gamma$ be a connected and co-connected strongly regular graph with parameters $(v, k, \lambda, \mu)$ and eigenvalues $k>r>s$. Then $\bar{\Gamma}$ is strongly regular with parameters $(v, v-k-1, v-2 k+\mu-2, v-2 k+\lambda)$ and eigenvalues $v-k-1>-s-1>-r-1$.

Finally we state some straightforward bounds for the parameters of strongly regular graphs.

Proposition 10. Let $\Gamma$ be a strongly regular graph with parameters $(v, k, \lambda, \mu)$. Then

(i) $v-2 k+\lambda \geqslant 0$ with equality if and only if $\Gamma$ is complete multipartite;

(ii) $k-\lambda-1 \geqslant 0$ with equality if and only if $\bar{\Gamma}$ is complete multipartite. 


\section{The clique adjacency polynomial}

Now we define our main tool, the clique adjacency polynomial. Given an edge-regular graph $\Gamma$ with parameters $(v, k, \lambda)$, define the clique adjacency polynomial $C_{\Gamma}(x, y)$ as

$$
C_{\Gamma}(x, y):=x(x+1)(v-y)-2 x y(k-y+1)+y(y-1)(\lambda-y+2) .
$$

The utility of the clique adjacency polynomial follows from [16, Theorem 1.1] (see also [17, Theorem 3.1]), giving:

Theorem 11. Let $\Gamma$ be an edge-regular graph with parameters $(v, k, \lambda)$. Suppose that $\Gamma$ has a clique of size $c \geqslant 2$. Then $C_{\Gamma}(b, c) \geqslant 0$ for all integers $b$.

As discussed in [16] and [17], Theorem 11 provides a way of bounding the clique number of an edge-regular graph using only its parameters. Indeed, by Theorem 11, for an edge-regular graph $\Gamma$ and some integer $c \geqslant 2$, if there exists an integer $b$ such that $C_{\Gamma}(b, c)<0$ then $\omega(\Gamma) \leqslant c-1$. Hence we define the clique adjacency bound (CAB) to be the least integer $c \geqslant 2$ such that $C_{\Gamma}(b, c+1)<0$ for some $b \in \mathbb{Z}$; note that such a $c$ always exists. Indeed, $C_{\Gamma}(b, y)$ is a cubic polynomial in $y$ with a negative leading coefficient.

We will show that, for a $k$-regular strongly regular graph $\Gamma$, the clique adjacency bound gives $\omega(\Gamma) \leqslant\lfloor 1-k / s\rfloor$. That is, the clique adjacency bound is always at least as good as the Delsarte bound when applied to strongly regular graphs. This follows from Theorem 11 together with Theorem 12 below. More interestingly, we will also show that the clique adjacency bound does better than the Delsarte bound for infinitely many feasible parameter tuples for strongly regular graphs. In this section we consider the univariate polynomial $C_{\Gamma}(f(t), g(t))$ in the variable $t$, where $f(t)$ and $g(t)$ are linear polynomials in $t$. The main idea is to choose the linear polynomials $f$ and $g$ such that there exists $t \in \mathbb{R}$ such that $C_{\Gamma}(f(t), g(t))<0, f(t) \in \mathbb{Z}$, and $g(t)$ is an integer at least 2 . We begin by stating one of the main results of this paper.

Theorem 12. Let $\Gamma$ be a strongly regular graph with parameters $(v, k, \lambda, \mu)$ and eigenvalues $k \geqslant r>s$. Then,

$$
C_{\Gamma}(\lfloor-\mu / s\rfloor,\lfloor 2-k / s\rfloor)<0 \text {. }
$$

Proof. Follows from Corollary 15 and Corollary 18 below.

Now we can state our first polynomial identity, which shows that the clique adjacency polynomial is negative at a certain point.

Lemma 13. Let $\Gamma$ be a connected strongly regular graph with parameters $(v, k, \lambda, \mu)$ and eigenvalues $k>r>s$. Then

$$
C_{\Gamma}(-\mu / s, 2-k / s)=(2 s-r)(r+1)<0 .
$$

Proof. The equality follows from direct calculation, using the equalities in Proposition 6 (see Appendix A). The right-hand side is negative since $s<0$ and $r \geqslant 0$. 
Let $\Gamma$ be a strongly regular graph with parameters $(v, k, \lambda, \mu)$ such that both $\mu / s$ and $k / s$ are integers. Then by Lemma 13, together with Theorem 11, we recover the Delsarte bound, i.e., $\omega(\Gamma) \leqslant\lfloor 1-k / s\rfloor$. It remains for us to deal with the situation when $k / s$ and $\mu / s$ are not integers. In the remainder of this section, motivated by Lemma 13, we consider integral points $(x, y) \in \mathbb{Z}^{2}$ close to $(-\mu / s, 2-k / s)$ such that $C_{\Gamma}(x, y)$ is negative. We deal with the type I and type II cases separately.

\subsection{Type-I strongly regular graphs}

Let $\Gamma$ be a type-I strongly regular graph (or conference graph) with $v$ vertices. By Proposition 7 we have $-\mu / s=r$ and $-k / s=2 r$. Therefore, we consider integral points $(x, y)$ close to $(r, 2+2 r)$ at which to evaluate the clique adjacency polynomial. In view of Proposition 8 , we evaluate $C_{\Gamma}(x, y)$ at points of the form $(r-t, a+2 r-2 t)$ for some $a \in \mathbb{N}$, thinking of $t$ as the fractional part of $r$.

Lemma 14. Let $\Gamma$ be a type-I strongly regular graph with $v$ vertices and eigenvalues $k>r>s$. Then

$$
\begin{aligned}
& C_{\Gamma}(r-t, 3+2 r-2 t)=2(t-1)(t+s-2)(t+2 s) \\
& C_{\Gamma}(r-t, 2+2 r-2 t)=(t+s)\left(2 t^{2}+(4 s-1) t-3 s-1\right) .
\end{aligned}
$$

Proof. The equalities follow from direct calculation, applying Proposition 6 and the definition of a type-I strongly regular graph (see Appendix A).

The right-hand side of Equation (2) is a cubic polynomial in the indeterminate $t$ with positive leading coefficient. Furthermore, since for a type-I strongly regular graph we have $s=-(\sqrt{v}+1) / 2$, we observe that the smallest zero of the right-hand side of Equation (2) is equal to $3 / 4-(\sqrt{v+5 / 4}-\sqrt{v}) / 2$. Hence $C_{\Gamma}(r-t, 2+2 r-2 t)$ is negative for $t<3 / 4-(\sqrt{v+5 / 4}-\sqrt{v}) / 2$. We use this observation in the next result, which can be used with Theorem 11 to obtain the Delsarte bound for conference graphs.

Corollary 15. Let $\Gamma$ be a type-I strongly regular graph with $v$ vertices. Then

$$
C_{\Gamma}(\lfloor(\sqrt{v}-1) / 2\rfloor,\lfloor\sqrt{v}+1\rfloor)<0 .
$$

Proof. Let $t=$ frac $(r)$. If $t>1 / 2$ then

$$
C_{\Gamma}(\lfloor(\sqrt{v}-1) / 2\rfloor,\lfloor\sqrt{v}+1\rfloor)=C_{\Gamma}(r-t, 3+2 r-2 t)
$$

and the right-hand side of Equation (1) is negative for $t<1$. Otherwise, if $t<1 / 2$ then

$$
C_{\Gamma}(\lfloor(\sqrt{v}-1) / 2\rfloor,\lfloor\sqrt{v}+1\rfloor)=C_{\Gamma}(r-t, 2+2 r-2 t),
$$

which is negative since $t<1 / 2<3 / 4-(\sqrt{v+5 / 4}-\sqrt{v}) / 2$. Note that $t$ cannot be equal to $1 / 2$ since $r$ is an algebraic integer.

The next corollary follows in a similar fashion. 
Corollary 16. Let $\Gamma$ be a type-I strongly regular graph with $v$ vertices. Suppose that

$$
0<\operatorname{frac}(\sqrt{v} / 2)<1 / 4-(\sqrt{v+5 / 4}-\sqrt{v}) / 2 .
$$

Then $C_{\Gamma}(\lfloor(\sqrt{v}-1) / 2\rfloor,\lfloor\sqrt{v}\rfloor)<0$.

Proof. Let $t=\operatorname{frac}(r)=\operatorname{frac}((\sqrt{v}-1) / 2)$. Then by our hypothesis $1 / 2<t<3 / 4-$ $(\sqrt{v+5 / 4}-\sqrt{v}) / 2$. Therefore we have

$$
C_{\Gamma}(\lfloor(\sqrt{v}-1) / 2\rfloor,\lfloor\sqrt{v}\rfloor)=C_{\Gamma}(r-t, 2+2 r-2 t),
$$

which is negative since $t<3 / 4-(\sqrt{v+5 / 4}-\sqrt{v}) / 2$.

\subsection{Type-II strongly regular graphs}

Let $\Gamma$ be a type-II strongly regular graph with parameters $(v, k, \lambda, \mu)$. Again, in view of Proposition 8 , we evaluate $C_{\Gamma}(x, y)$ at points of the form $(-\mu / s-t, a-k / s-t)$ for some $a \in \mathbb{Z}$, thinking of $t$ as the fractional part of $-\mu / s$.

Lemma 17. Let $\Gamma$ be a strongly regular graph with parameters $(v, k, \lambda, \mu)$ and eigenvalues $k \geqslant r>s$. Then

$$
\begin{aligned}
& C_{\Gamma}(-\mu / s-t, 2-k / s-t)=(t-1)((v-2 k+\lambda) t-(2 s-r)(r+1)) \\
& C_{\Gamma}(-\mu / s-t, 1-k / s-t)=t((v-2 k+\lambda)(t-1)+r(r+1)) .
\end{aligned}
$$

Moreover, if $\Gamma$ is co-connected then these polynomials have positive leading coefficients.

Proof. The equalities follow from direct calculation, using the equalities in Proposition 6 (see Appendix A). By Proposition 10 if $\Gamma$ is co-connected then the polynomials have positive leading coefficients.

Note that Lemma 13 is a special case of Lemma 17, for $t=0$.

Corollary 18. Let $\Gamma$ be a type-II strongly regular graph with parameters $(v, k, \lambda, \mu)$ and eigenvalues $k \geqslant r>s$. Then

$$
C_{\Gamma}(\lfloor-\mu / s\rfloor,\lfloor 2-k / s\rfloor)<0 .
$$

Proof. If $\Gamma$ is disconnected then we have $\mu=0$ and $2-k / s=\lambda+3$. Whence we have $C_{\Gamma}(\lfloor-\mu / s\rfloor,\lfloor 2-k / s\rfloor)=C_{\Gamma}(0, \lambda+3)=-(\lambda+3)(\lambda+2)<0$, as required. Hence we can assume that $\Gamma$ is connected.

Let $t=\operatorname{frac}(-\mu / s)$. Then, using Proposition 8 and Equation (3), we have

$$
C_{\Gamma}(\lfloor-\mu / s\rfloor,\lfloor 2-k / s\rfloor)=(t-1)((v-2 k+\lambda) t-(2 s-r)(r+1)) .
$$

Suppose first that $\Gamma$ is co-connected. The right-hand side of Equation (3) is negative on the open interval $(\eta, 1)$, where $\eta=(2 s-r)(r+1) /(v-2 k+\lambda)$ is negative. Hence the corollary holds for $\Gamma$. On the other hand, for complete multipartite graphs we have $t=0$, in which case the right-hand side of Equation (3) is negative. 
The next corollary follows similarly, using the fact that the right-hand side of Equation (4) is negative on the open interval $(0, \eta)$, where $\eta=1-\left(r^{2}+r\right) /(v-2 k+\lambda)$.

Corollary 19. Let $\Gamma$ be a co-connected type-II strongly regular graph with parameters $(v, k, \lambda, \mu)$ and eigenvalues $k \geqslant r>s$. Suppose that

$$
0<\operatorname{frac}(-k / s)<1-\left(r^{2}+r\right) /(v-2 k+\lambda) .
$$

Then $C_{\Gamma}(\lfloor-\mu / s\rfloor,\lfloor 1-k / s\rfloor)<0$.

Remark 20. We remark that if a type-II strongly regular graph satisfies the hypothesis of Corollary 19 then its complement cannot also satisfy the hypothesis. Indeed, suppose that $\Gamma$ satisfies the hypothesis of Corollary 19. Since frac $(-k / s)>0$ we have that $s \neq-1$ and hence $\Gamma$ is connected. Then, using Proposition 9, we see that the complement of $\Gamma$ also satisfies the hypothesis of Corollary 19 if

$$
0<\operatorname{frac}((v-k-1) /(r+1))<1-\left(s^{2}+s\right) / \mu .
$$

In particular, for the corollary to hold for both $\Gamma$ and its complement, we must have both $\left(r^{2}+r\right) /(v-2 k+\lambda)<1$ and $\left(s^{2}+s\right) / \mu<1$. But we find that $\left(r^{2}+r\right) /(v-2 k+\lambda)<1$ if and only if $\left(s^{2}+s\right) / \mu>1$. One can see this by using the equalities in Proposition 6 to obtain the equality

$$
\mu(v-2 k+\lambda)=\left(r^{2}+r\right)\left(s^{2}+s\right)
$$

\section{$5 \quad$ How sharp is the clique adjacency bound?}

In this section we show that the clique adjacency bound is sharp for strongly regular graphs in certain instances. We also comment on the sharpness of the clique adjacency bound for general strongly regular graphs.

Theorem 21. Let $\Gamma$ be a strongly regular graph with parameters $(v, k, \lambda, \mu)$ and eigenvalues $k \geqslant r>s$. Suppose that $\lambda+1 \leqslant-k / s$. Then the clique adjacency bound is equal to $\lambda+2$.

As the second author observed in [17], for an edge-regular graph with parameters $(v, k, \lambda)$ we have $C_{\Gamma}(0, y)=-y(y-1)(y-(\lambda+2))$, so for all $y>\lambda+2$, we have $C_{\Gamma}(0, y)<0$. Hence the clique adjacency bound is always at most the trivial bound of $\lambda+2$. Therefore, to prove Theorem 21, it suffices to show that, for strongly regular graph parameters satisfying $\lambda+1 \leqslant-k / s$, the clique adjacency bound is at least $\lambda+2$.

Lemma 22. Let $\Gamma$ be a connected type-II strongly regular graph with parameters $(v, k, \lambda, \mu)$ and eigenvalues $k>r>s$. Suppose that $\lambda+1 \leqslant-k / s$. Then $C_{\Gamma}(1, \lambda+2) \geqslant 0$ with equality if and only if $\lambda=-k / s-1$. 
Proof. First suppose $\lambda+1=-k / s$. Using the equations $r=\lambda-\mu-s$ and $r=(\mu-k) / s$ from Proposition 6, we find that

$$
\lambda-\mu-s=-k / s-1-\mu-s=(\mu-k) / s .
$$

From this it follows that $-\mu / s=1$. In this case, $C_{\Gamma}(1, \lambda+2)=C_{\Gamma}(-\mu / s, 1-k / s)$, which is zero by Equation (4).

It remains to assume $\lambda+1<-k / s$. Using Proposition 6 , we can write

$$
\frac{\mu}{2} C_{\Gamma}(1, \lambda+2)=k(k-(\mu+1)(\lambda+1))+\mu(\lambda+1)^{2} .
$$

To show this quantity is positive, it suffices to show that $k-(\mu+1)(\lambda+1)$ is positive. Using the inequality $\lambda+1<-k / s$, we have $k-(\mu+1)(\lambda+1)>k(1+(\mu+1) / s)$. It therefore suffices to show that $1+(\mu+1) / s \geqslant 0$.

Since $\lambda=k+r+s+r s$, the inequality $\lambda+1<-k / s$ becomes

$$
k+(r+1)(s+1)<-k / s .
$$

Since $s<-1$, it follows that $r+1>-k / s$. Multiplying this inequality by $-s$ gives $-s(r+1)>k$. Since both $s$ and $r$ are integers, we have $-s(r+1) \geqslant k+1$. Now by rearranging and substituting $\mu=k+r s$, we obtain the inequality $1+(\mu+1) / s \geqslant 0$ as required.

Lemma 23. Let $\Gamma$ be an edge-regular graph with parameters $(v, k, \lambda)$ such that $C_{\Gamma}(b, \lambda+$ $2) \geqslant 0$ for all integers $b$. Then $C_{\Gamma}(b, c) \geqslant 0$ for all $c \in\{2, \ldots, \lambda+2\}$ and all integers $b$.

Proof. Let $c \in\{2, \ldots, \lambda+2\}$ and let $b$ be an integer. If $b \leqslant 0$, then from the definition of the clique adjacency polynomial $C_{\Gamma}(x, y)$, we see that $C_{\Gamma}(b, c) \geqslant 0$, so we now assume that $b$ is positive.

A calculation (see Appendix A) shows that

$$
C_{\Gamma}(b, c)-C_{\Gamma}(b, \lambda+2)=(\lambda+2-c)(b-c)(b-c+1)+2 b(\lambda+2-c)(k-\lambda-1) .
$$

This quantity is nonnegative since $b$ and $\lambda+2-c$ are nonnegative integers, the product of two consecutive integers is nonnegative, and $k-\lambda-1$ is also nonnegative by Proposition 10 . Hence

$$
C_{\Gamma}(b, c) \geqslant C_{\Gamma}(b, \lambda+2) \geqslant 0,
$$

as required.

Now we prove Theorem 21.

Proof of Theorem 21. Firstly, if $\Gamma$ is disconnected then $\Gamma$ is the disjoint union of complete graphs and hence contains cliques of size $\lambda+2$. Therefore the clique adjacency bound is at least $\lambda+2$. Now we assume that $\Gamma$ is connected. 
By Lemma 23, the clique adjacency bound is less than $\lambda+2$ only if there exists some integer $b$ such that $C_{\Gamma}(b, \lambda+2)$ is less than zero. To ease notation set $f(x):=C_{\Gamma}(x, \lambda+2)$. Hence

$$
f(x)=x((v-\lambda-2) x+v+(2 \lambda-2 k+1)(\lambda+2)) .
$$

It suffices to show that there does not exist any integer $b$ such that $f(b)<0$.

Observe that the polynomial $f(x)$ is a quadratic polynomial in the variable $x$. Furthermore, the leading coefficient of $f(x)$ is $v-\lambda-2 \geqslant 0$, and $f(0)=0$. Let $\xi$ be the other zero of $f(x)$. Now, $f(x)$ is negative if and only if $x$ is between 0 and $\xi$. Hence, if $f(-1)$ and $f(1)$ are both nonnegative then there are no integers $b$ such that $f(b)<0$. As in the proof of the previous result $f(-1)$ is nonnegative. Therefore Lemma 22 completes the proof for type-II strongly regular graphs.

The inequality $\lambda+1 \leqslant-k / s$ only holds for type-I strongly regular graphs on 5 vertices or 9 vertices (where we have equality). One can explicitly compute the clique adjacency bound for these two cases: the unique $(5,2,0,1)$-strongly regular graph and the unique $(9,4,1,2)$-strongly regular graph. For each of these graphs the clique adjacency bound is equal to $\lambda+2$.

Now we give a couple of remarks about Theorem 21 .

Remark 24. For strongly regular graphs with $\lambda \leqslant 1$, it is easy to see that the clique number is $\lambda+2$. By Theorem 21, the clique adjacency bound is equal to the clique number for such graphs. Let $\Gamma$ be a strongly regular graph with parameters $(v, k, \lambda, \mu)$. By Proposition 6, we see that $k=-s(r+1)-r+\lambda$. Therefore, for strongly regular graphs with $\lambda=2$ and $r \geqslant 2$, we have $\lambda+1=3 \leqslant-k / s$, and so Theorem 21 applies to such graphs.

Remark 25. We conjecture that if the clique adjacency bound is less than $-k / s$ then $\lambda+1 \leqslant-k / s$. We have verified this conjecture for all feasible parameter tuples for strongly regular graphs on up to 1300 vertices, making use of Brouwer's website [7].

In Table 1, we list all the feasible parameter tuples for strongly regular graphs on at most 150 vertices to which we can apply either Theorem 1 or Theorem 4 . In other words, Table 1 displays the feasible parameters for strongly regular graphs on at most 150 vertices for which the clique adjacency bound is strictly less than the Delsarte bound. In the column labelled 'Exists', if there exists a strongly regular graph with the appropriate parameters then we put ' + ', or '!' if the graph is known to be unique; otherwise, if the existence is unknown, we put '?'. In the final column of Table 1, we put ' $\mathrm{Y}$ ' (resp. ' $N$ ') if there exists (resp. does not exist) a strongly regular graph with the corresponding parameters that has clique number equal to the clique adjacency bound, otherwise we put a '?' if such existence is unknown. We refer to Brouwer's website [7] for details on the existence of strongly regular graphs with given parameters.

For the parameter tuples in Table 1, the Delsarte bound is equal to the clique adjacency bound plus 1. As an example of a parameter tuple for which the clique adjacency bound differs from the Delsarte bound by 2 , we have $(378,52,1,8)$ for which there exists a corresponding graph [11]. For this graph the Delsarte bound is 5, but the clique adjacency bound is 3 . 


\begin{tabular}{l|c|c|c|c} 
Parameters & Type & CAB & Exists & Sharp \\
\hline$(17,8,3,4)$ & I & 3 & $!$ & Y \\
$(37,18,8,9)$ & I & 5 & + & Y \\
$(50,7,0,1)$ & II & 2 & $!$ & Y \\
$(56,10,0,2)$ & II & 2 & $!$ & Y \\
$(65,32,15,16)$ & I & 7 & $?$ & $?$ \\
$(77,16,0,4)$ & II & 2 & $!$ & Y \\
$(88,27,6,9)$ & II & 4 & $?$ & $?$ \\
$(99,14,1,2)$ & II & 3 & $?$ & Y \\
$(100,22,0,6)$ & II & 2 & $!$ & Y \\
$(101,50,24,25)$ & I & 9 & + & $?$ \\
$(105,32,4,12)$ & II & 3 & $!$ & Y \\
$(111,30,5,9)$ & II & 4 & $?$ & $?$ \\
$(115,18,1,3)$ & II & 3 & $?$ & Y \\
$(120,42,8,18)$ & II & 3 & $!$ & Y \\
$(121,36,7,12)$ & II & 4 & $?$ & $?$ \\
$(133,32,6,8)$ & II & 5 & $?$ & $?$ \\
$(144,39,6,12)$ & II & 4 & + & Y \\
$(144,52,16,20)$ & II & 6 & $?$ & $?$ \\
$(145,72,35,36)$ & I & 11 & $?$ & $?$ \\
$(149,74,36,37)$ & I & 11 & + & $?$
\end{tabular}

Table 1: Feasible parameter tuples for strongly regular graphs on at most 150 vertices to which we can apply either Theorem 1 or Theorem 4 .

Although strongly regular graphs with parameters $(16,10,6,6)$ and $(27,16,10,8)$ exist, there are no strongly regular graphs with these parameters whose clique number is equal to the clique adjacency bound. Thus, we ask the following question. Do there exist strongly regular graphs with parameters $(v, k, \lambda, \mu)$, with $k<v / 2$, such that every strongly regular graph having those parameters has clique number less than the clique adjacency bound?

\section{Hoffman bound vs Delsarte bound vs clique adjacency bound}

Let $\Gamma$ be a non-complete regular graph with $v$ vertices, valency $k$, and second largest eigenvalue $r \leqslant k$. Then the complement $\bar{\Gamma}$ of $\Gamma$ is a regular graph with valency $\bar{k}=v-k-1$ and least eigenvalue $\bar{s}=-r-1<0$. We may obtain a bound for the clique number of $\Gamma$ by applying the Hoffman bound (also called the ratio bound) [13, Theorem 2.4.1] on the size of a largest independent set (coclique) of $\bar{\Gamma}$. This gives

$$
\omega(\Gamma) \leqslant\left\lfloor\frac{v}{1-\bar{k} / \bar{s}}\right\rfloor
$$

If $\Gamma$ is strongly regular, then it is known (and follows from the relations of Proposition 6) that the Delsarte bound for $\omega(\Gamma)$ is the same as that given by the Hoffman bound above. 
Now the Delsarte bound applies not only to strongly regular graphs, but also to the graphs $\left\{\Gamma_{1}, \ldots, \Gamma_{d}\right\}$ of the relations (other than equality) of any $d$-class symmetric association scheme (see [13, Corollary 3.7.2]). Thus, if $\Gamma$ is such a graph, having valency $k$ and least eigenvalue $s$, then $\omega(\Gamma) \leqslant\lfloor 1-k / s\rfloor$.

Here is an interesting illustrative example. Let $\Delta$ be the edge graph (or line graph) of the incidence graph of the projective plane of order 2 . Then $\Delta$ is the unique distanceregular graph with intersection array $\{4,2,2 ; 1,1,2\}$. Now let $\Delta_{3}$ be the graph on the vertices of $\Delta$, with two vertices joined by an edge if and only if they have distance 3 in $\Delta$. Then $\Delta_{3}$ is the graph of a relation in the usual symmetric association scheme associated with a distance-regular graph, where two vertices are in relation $i$ precisely when they are at distance $i$ in the distance-regular graph. The graph $\Delta_{3}$ has diameter 2 and is edge-regular (but not strongly regular) with parameters $(v, k, \lambda)=(21,8,3)$. The clique adjacency bound for $\Delta_{3}$ is 4 . The least eigenvalue of $\Delta_{3}$ is $-\sqrt{8}$, and the Delsarte bound gives 3 , and indeed, $\omega\left(\Delta_{3}\right)=3$. However, the complement of $\Delta_{3}$ has least eigenvalue $-1-\sqrt{8}$, and the Hoffman bound for independent sets in the complement of $\Delta_{3}$ gives 5. Thus, for $\Delta_{3}$, the Delsarte bound is better than the clique adjacency bound which is better than that obtained from the Hoffman bound. However, the three bounds are for different classes of graphs. For example, there may well be an edge-regular graph with parameters $(21,8,3)$ and clique number 4 . It would be interesting to find one.

We conjecture that if $\Gamma$ is any non-complete edge-regular graph, then the clique adjacency bound for $\omega(\Gamma)$ is at most that obtained from the Hoffman bound for $\bar{\Gamma}$.

Since we have proved that for a strongly regular graph the clique adjacency bound is at most the Delsarte bound, the conjecture is true when $\Gamma$ is strongly regular. The conjecture has also been verified computationally by Rhys Evans for all vertex-transitive edge-regular graphs having at most 47 vertices, using lists of such graphs computed by Derek Holt and Gordon Royle [14]. Furthermore, the conjecture is true when $\Gamma$ is disconnected. Indeed, suppose $\Gamma$ is a disconnected edge-regular graph with parameters $(v, k, \lambda)$. Then $\Gamma$ has largest eigenvalue $k$ with multiplicity at least 2 , so the complement of $\Gamma$ has least eigenvalue $-k-1$. Hence, the Hoffman bound for this complement is $v /(1+(v-k-$ $1) /(k+1))=k+1$, which is greater than or equal to $\lambda+2$, which in turn is greater than or equal to the clique adjacency bound for $\Gamma$.

\section{A Algebraic computational verification of identities}

In this appendix we present the algebraic computations in Maple [3] that were used to verify certain identities employed in this paper. These identities were also checked independently using Magma [5].

We start up Maple (version 18) and assign to $C$ the clique adjacency polynomial.

$>\mathrm{C}:=\mathrm{x} *(\mathrm{x}+1) *(\mathrm{v}-\mathrm{y})-2 * \mathrm{x} * \mathrm{y} *(\mathrm{k}-\mathrm{y}+1)+\mathrm{y} *(\mathrm{y}-1) *(1 \mathrm{ambda}-\mathrm{y}+2):$

We then make a set of relators, obtained from Proposition 6, which evaluate to 0 on the parameters $(v, k, \lambda, \mu)$ and eigenvalues $r, s$ (with $k \geqslant r>s$ ) of a strongly regular graph. 
$>$ srg_rels $:=\{(v-k-1) * m u-k *(k-l a m b d a-1),(l a m b d a-m u)-(r+s),(m u-k)-r * s\}:$

We make a further set of relators which evaluate to 0 on the parameters and eigenvalues of a type-I strongly regular graph.

$>$ type1_rels:=srg_rels union $\{2 * \mathrm{k}-(\mathrm{v}-1), 4 * \mathrm{lambda}-(\mathrm{v}-5), 4 * \mathrm{mu}-(\mathrm{v}-1)\}$ :

Let $R=\mathbb{Q}[t, v, k, \lambda, \mu, r, s]$ be the ring of polynomials over $\mathbb{Q}$ in the indeterminates $t, v, k, \lambda, \mu, r, s$, let $S$ be the ideal of $R$ generated by srg_rels given above, and let $T$ be the ideal of $R$ generated by type1_rels. We use the Maple package Groebner to calculate and employ Gröbner bases [10] to work in the factor rings $R / S$ and $R / T$.

We set the monomial ordering for the Gröbner basis calculations to be the Maple tdeg ordering, more commonly called the grevlex ordering, with the indeterminates ordered as $t>v>k>\lambda>\mu>r>s$.

$>\operatorname{ordering}:=\operatorname{tdeg}(\mathrm{t}, \mathrm{v}, \mathrm{k}, \mathrm{lambda}, \mathrm{mu}, \mathrm{r}, \mathrm{s})$ :

Then we compute a Gröbner basis $G$ for $S$.

$>\mathrm{G}:=$ Groebner [Basis] (srg_rels, ordering):

For the record, $G=\left[\lambda-\mu-r-s, r s+k-\mu, k^{2}-k r-k s-\mu v-k+\mu\right]$. Similarly, we compute a Gröbner basis $H$ for $T$.

$>\mathrm{H}:=$ Groebner [Basis] (type1_rels, ordering) :

Here, we obtain $H=\left[r+1+s, \lambda+1-\mu, k-2 \mu, v-1-4 \mu, s^{2}-\mu+s\right]$.

We now verify that the identity of Lemma 13 holds, by checking that $s^{3}(C(-\mu / s, 2-$ $k / s)-(2 s-r)(r+1))=0$ in $R / S$.

$>$ Groebner [NormalForm] (expand ( $\mathrm{s}^{\wedge} 3 *(\mathrm{eval}(\mathrm{C},[\mathrm{x}=-\mathrm{mu} / \mathrm{s}, \mathrm{y}=2-\mathrm{k} / \mathrm{s}])$

$>\quad-(2 * s-r) *(r+1))), G$, ordering $)$;

0

Similarly, we verify that the identities of Lemma 14 hold for type-I strongly regular graphs, by working in $R / T$.

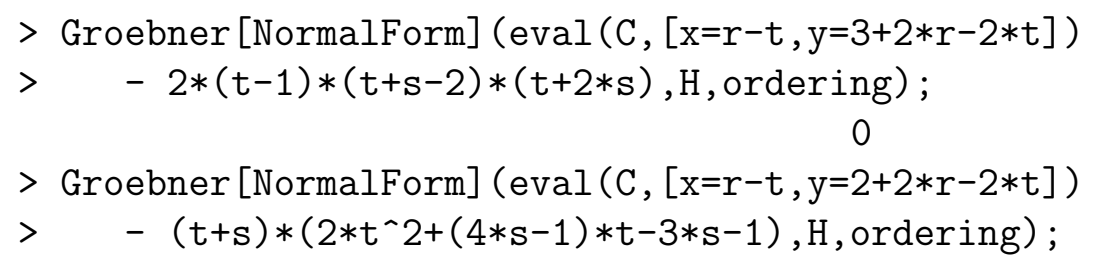


Next are the verifications of the identities of Lemma 17.

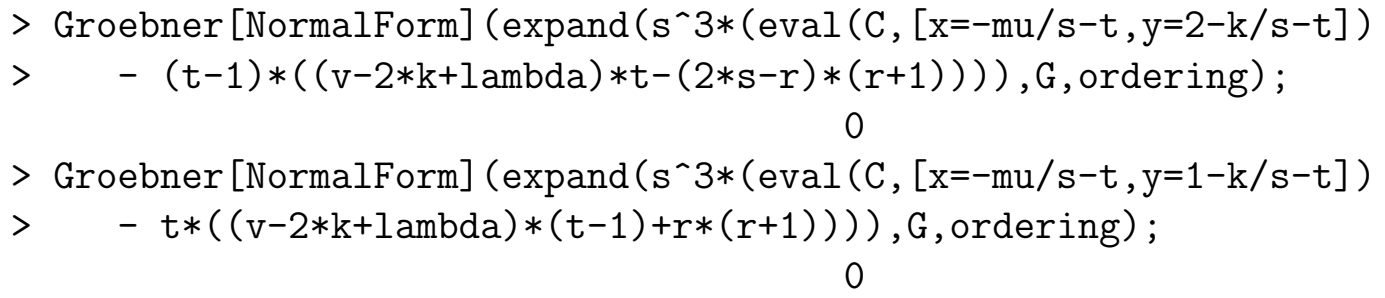

Here is confirmation of the identity used in Remark 20.

$>$ Groebner [NormalForm] (mu*(v-2*k+lambda) $-\left(r^{\wedge} 2+r\right) *\left(s^{\wedge} 2+s\right), G$, ordering $)$;

0

Next is verification of the identity used in the proof of Lemma 22.

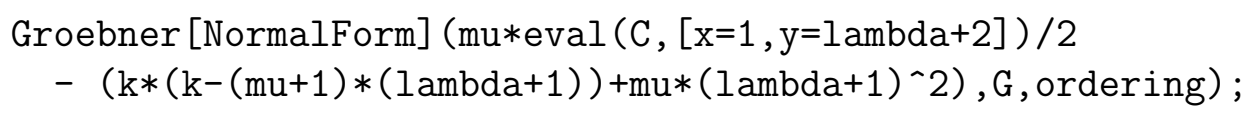

0

Finally, here is a confirmation of the polynomial equality used in the proof of Lemma 23.

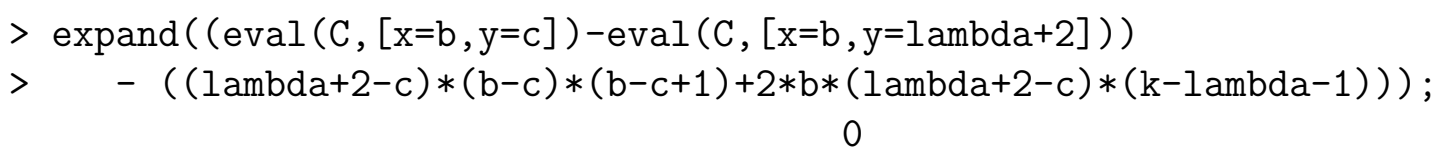

\section{Acknowledgement}

We thank Anton Betten for organising the Combinatorics and Computer Algebra 2015 conference, whose problem sessions brought us together to begin this work.

\section{References}

[1] C. Bachoc, M. Matolcsi, and I. Z. Ruzsa. Squares and difference sets in finite fields. Integers, 13:A77, 2013.

[2] A. Balog. On the distribution of $p^{\theta} \bmod 1$. Acta Math. Hungar., 45(1-2):179-199, 1985.

[3] L. Bernardin, P. Chin, P. DeMarco, K. O. Geddes, D. E. G. Hare, K. M. Heal, G. Labahn, J. P. May, J. McCarron, M. B. Monagan, D. Ohashi, and S. M. Vorkoetter. Maple Programming Guide. Maplesoft, Waterloo ON, Canada, 2014. 
[4] A. Blokhuis. On subsets of $\mathrm{GF}\left(q^{2}\right)$ with square differences. Nederl. Akad. Wetensch. Indag. Math., 46(4):369-372, 1984.

[5] W. Bosma, J. Cannon, and C. Playoust. The Magma algebra system. I. The user language. J. Symbolic Comput., 24(3-4):235-265, 1997. Computational algebra and number theory (London, 1993).

[6] A. E. Brouwer. Strongly regular graphs. In C. J. Colbourn and J. H. Dinitz, editors, Handbook of Combinatorial Designs, Discrete Mathematics and its Applications (Boca Raton). Chapman \& Hall/CRC, Boca Raton, FL, second edition, 2007.

[7] A. E. Brouwer. http://www.win.tue.nl/ aeb/graphs/srg/srgtab.html, 2016.

[8] A. E. Brouwer and W. H. Haemers. Spectra of Graphs. Universitext, Springer, New York (2012), 2012.

[9] P. J. Cameron and J. H. van Lint. Designs, Graphs, Codes, and their Links. Cambridge University Press, 1992.

[10] A. Cohen. Gröbner bases, an introduction. In A. Cohen, H. Cuypers, and H. Sterk, editors, Some Tapas of Computer Algebra, pages 1-33. Springer, Berlin-HeidelbergNew York, 1999.

[11] A. Cossidente and T. Penttila. Hemisystems on the Hermitian surface. J. London Math. Soc. (2), 72(3):731-741, 2005.

[12] P. Delsarte. An algebraic approach to the association schemes of coding theory. $\mathrm{PhD}$ thesis, Universite Catholique de Louvain., 1973.

[13] C. Godsil and K. Meagher. Erdös-Ko-Rado Theorems: Algebraic Approaches. Cambridge University Press, 2016.

[14] D. Holt and G. Royle. Small transitive groups and vertex-transitive graphs. In preparation.

[15] L. Kuipers and H. Niederreiter. Uniform Distribution of Sequences. Wiley Interscience, New York, 1974.

[16] L. H. Soicher. More on block intersection polynomials and new applications to graphs and block designs. J. Combin. Theory Ser. A, 117(7):799-809, 2010.

[17] L. H. Soicher. On cliques in edge-regular graphs. J. Algebra, 421:260-267, 2015. 